\title{
Genetic manipulation of longevity-related genes as a tool to regulate yeast life span and metabolite production during winemaking
}

Helena Orozco ${ }^{1,2}$, Emilia Matallana ${ }^{1,2}$ and Agustín Aranda ${ }^{1 *}$

\begin{abstract}
Background: Yeast viability and vitality are essential for different industrial processes where the yeast Saccharomyces cerevisiae is used as a biotechnological tool. Therefore, the decline of yeast biological functions during aging may compromise their successful biotechnological use. Life span is controlled by a variety of molecular mechanisms, many of which are connected to stress tolerance and genomic stability, although the metabolic status of a cell has proven a main factor affecting its longevity. Acetic acid and ethanol accumulation shorten chronological life span (CLS), while glycerol extends it.

Results: Different age-related gene classes have been modified by deletion or overexpression to test their role in longevity and metabolism. Overexpression of histone deacetylase SIR2 extends CLS and reduces acetate production, while overexpression of SIR2 homolog HST3 shortens CLS, increases the ethanol level, and reduces acetic acid production. HST3 overexpression also enhances ethanol tolerance. Increasing tolerance to oxidative stress by superoxide dismutase SOD2 overexpression has only a moderate positive effect on CLS. CLS during grape juice fermentation has also been studied for mutants on several mRNA binding proteins that are regulators of gene expression at the posttranscriptional level; we found that NGR1 and UTH4 deletions decrease CLS, while PUF3 and PUB1 deletions increase it. Besides, the pub1D mutation increases glycerol production and blocks stress granule formation during grape juice fermentation. Surprisingly, factors relating to apoptosis, such as caspase Yca1 or apoptosis-inducing factor Aif1, play a positive role in yeast longevity during winemaking as their deletions shorten CLS.

Conclusions: Manipulation of regulators of gene expression at both transcriptional (i.e., sirtuins) and posttranscriptional (i.e., mRNA binding protein Pub1) levels allows to modulate yeast life span during its biotechnological use. Due to links between aging and metabolism, it also influences the production profile of metabolites of industrial relevance.
\end{abstract}

Keywords: Yeast, Chronological aging, Stress, Ethanol, Sirtuins, HST3, PUB1

\section{Background}

Biological aging, or senescence, is defined as the degradation of the biological function that accompanies the passage of time [1], and it is a process that affects cells, individuals and populations. Therefore, the performance of microorganisms during their biotechnological use can be affected by the life span of the members of a population for a given condition. For instance, baker's yeast

\footnotetext{
* Correspondence: arandaa@iata.csic.es

'Departamento de Biotecnología, Instituto de Agroquímica y Tecnología de Alimentos-CSIC, Av. Agustín Escardino, 7, Paterna 46980, Spain Full list of author information is available at the end of the article
}

Saccharomyces cerevisiae has two aging models [2]. Replicative life span (RLS) is the number of daughter cells produced by a mother cell before senescence, which can be easily visualized due to the asymmetric nature of $S$. cerevisiae cell divisions. This fixed amount of cell divisions becomes relevant when there is continuous growth, for instance during biomass propagation, beer production [3] or sugar cane fermentation to obtain biofuel [4], where the yeast biomass produced at the end of the processes is re-used to inoculate new fermentations. Chronological life span (CLS) is defined by how long a yeast cell can survive in a non dividing,

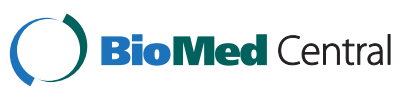


quiescence-like state. This aging model is more relevant when fermentation is carried out mostly by non dividing cells, which is the case of grape juice fermentation in winemaking [5]. Modern winemaking practices include inoculation of grape juice with starter cultures in the form of active dry yeasts. Under these conditions, the yeast growth phase implies only 4-6 cycles of cell division, far from the 20 divisions of the mean maximal RLS of natural isolates [6]. Therefore RLS is not a limiting factor for yeast performance, unlike viability in the stationary phase which is 3-4 times longer than the growth phase under winemaking conditions [5]. "Sur lies" aging refers to aging wine on yeast lees (death cells). During this period, cells undergo autolysis by releasing enzymes that change the wine composition to generate desirable organoleptic properties [7]. Release of intracellular components after cell death and lysis may also influence the growth of microorganisms, and they may be positive for winemaking, such as lactic acid bacteria involved in malolactic fermentation [8], or negative; e.g., growth of spoiling microorganisms, such as other yeasts or acetic bacteria. The environmental factors involved in CLS during winemaking have been studied in our laboratory, and it is clear that the high concentration of two-carbon metabolites produced by yeast metabolism, such as ethanol, acetic acid and acetaldehyde, are key factors for longevity [9].

The traditional biochemical way of describing senescence has been the free radical theory of aging, established in 1956 [10]. Relevance of the oxygen reactive species generated by metabolism or by exogenous oxidants on life span has been described in many organisms, including yeast [11]. In a previous work, we demonstrated that tolerance to oxidative stress correlates to CLS in wine yeasts [12]. However, there is an increasing challenge for this conventional conception of aging, and many authors interpret oxidative damage as a consequence, and not a cause, of aging [13].

In any case, it is clear that aging is a complex process involving a variety of molecular mechanisms, many of which have been discovered in yeast [2]. The first screening for yeast mutants with increased RLS identified four genes known as UTH1-4 [14]. One of them, $U T H 4$, codes for an RNA binding protein related to the re-localization of another aging protein, Uth2, from the telomere to the nucleolus [15]. Uth2 happens to be Sir4, a member of a complex ligated to genomic stability in which sirtuin Sir2 plays an important role. Sirtuins are $\mathrm{NAD}^{+}$-dependent histone deacetylases [16]. There are five yeast sirtuins, Sir2 (which gives its name to the family) and Hst1-4. Sir2 has been defined as an RLS extending factor given its role in heterochromatin formation, therefore promoting genome stability. The silencing of sub-telomeric regions by the action of Sir2 seems to be a key factor in replicative life span [17]. However, the role of Sir2 in CLS seems to be the opposite as Sir2 blocks CLS by repressing the activity of alcohol dehydrogenase 2 , therefore increasing the ethanol concentration in the stationary phase, which acts a pro-aging factor [18]. It has been reported that direct acetylation/deacetylation is a posttranslational form of metabolic regulation. In yeast, the regulation of the gluconeogenic Pck1 enzyme by Sir2 has been fully described [19]. The fact that this family of deacetylases consumes a $\mathrm{NAD}^{+}$molecule in their enzymatic reaction makes them act as metabolic sensors, and global analyses suggest that Sir2 controls a complex metabolic network [20]. In addition to ethanol, two other metabolites produced during fermentation have been linked to CLS under laboratory conditions. Acidification by acetic acid production is a very important cause of aging in laboratory conditions [21,22], while glycerol accumulation extends longevity [23].

A consensus has been reached that low food intake without malnutrition, i.e. dietary restriction (DR), is the only intervention that extends life span from yeast to mammals [24]. Sir2 has been described as a key factor in extending RLS by glucose restriction [25]. However, this hypothesis has been recently challenged and it is now the center of bitter controversy [26]. Moreover, the role of Sir2 overexpression in aging in flies and worms has proved not to be as strong as previously assumed [27]. Previously, we proved that sirtuin deletion in wine yeasts affects CLS, often in a growth medium-dependent way [28]. For instance, Sir2 deletion extends CLS under laboratory conditions, but shortens it during grape juice fermentation. Besides, sirtuin deletion affects metabolite production during winemaking as $\operatorname{sir} 2 \Delta$ mutants produce more ethanol and less acetic acid, while hst $3 \Delta$ has the opposite effect. Therefore, sirtuin manipulation can be envisaged as an efficient way to control metabolism.

Yeast death during chronological aging under laboratory conditions has been related to similar features to programmed cell death or apoptosis in higher organisms [29]. The benefits of apoptosis on single cell microorganisms have to be considered in the population survival context. The "altruistic aging" concept suggests that some cells may die and then nutrients to the rest of the colony are released. In this kind of aging, the oxidative stress caused by the superoxide anion is important, while the action of superoxide dismutases, like Sod2, blocks its action [30]. Many molecular components in apoptotic machinery are present in S. cerevisiae, such as caspase Yca1 and mitochondrial apoptosis-inducing factor Aif1 [31].

Our aim in this work was to manipulate a variety of genetic determinants of cell aging in order to study their influence on cell viability during grape juice fermentation and their impact on metabolism under this 
condition. The overexpressions of sirtuins SIR2 and HST3 prove to be an efficient tool to manipulate longevity and metabolite production. Increasing doses of HST3 produce more ethanol and less acetic acid, while the overexpression of SIR2 extends longevity. Manipulation of the oxidative stress machinery represented by the gene coding for superoxide dismutase 2 has only a moderate impact on life span, while deletion of apoptosis factors unexpectedly shortened CLS. We studied the role of several mRNA binding proteins as potential posttranscriptional regulators, and identified PUB1 as the gene whose deletion increases both CLS and glycerol production under winemaking conditions. Therefore, life span is closely linked to metabolism during grape juice fermentation by wine yeasts.

\section{Results and discussion}

\section{Modulation of life span by the overexpression of sirtuin} genes

In order to test the impact of sirtuin overexpression under winemaking conditions, the SIR2 gene was expressed under the control of two heterologous promoters following the promoter-replacement strategy developed in our laboratory [32]. Two promoters with different expression profiles during grape juice fermentation [33] were chosen. SPI1 is a stationary phase specific gene that has been described to be induced at late fermentation stages [33,34]. MET17 is a gene involved in methionine biosynthesis which is activated during the first days of fermentation [33]. Overexpression was performed in wine yeast strain L2056, a diploid for the SIR2 gene [28], by promoter replacement on one of its two copies. Transformants were used to perform natural grape juice fermentation, and yeast proteins were extracted 1 or 5 days after inoculation for Sir2 detection by using a specific antibody (Figure 1A). On day 1 , the SPI1 promoter was repressed if compared to the parental strain, but the MET17 promoter caused a higher SIR2 expression, as expected. On day 5, when cells have entered stationary phase (see Figure 1B) and less than one fourth of initial sugars were present, SPI1 led to a higher Sir2 expression than in the wild-type strain, as expected. Unexpectedly however, MET17 also accomplished this, indicating that, under our conditions, either this gene showed a high expression throughout fermentation or high levels of the Sir2 protein accumulated at the beginning of fermentation to remain stable for a long time. In any case, the overexpression strategy via promoter replacement rendered the expected result of increasing Sir2 levels. Hence, it is a successful way of increasing the dose of selected proteins during grape juice fermentation on wine yeast.

Next we analyzed the evolution of natural grape juice fermentation carried out by the constructed strains
(Figure 1B). Growth and death were monitored by counting the viable cells able to form a colony when plated in rich medium and expressed as cfu (colony forming units) per $\mathrm{mL}$. Fermentation progress was followed by measuring the amount of remaining sugars. All the manipulated strains were able to finish fermentation and to consume all the sugars in a similar time. Therefore, their fermentative capacity was not challenged. SIR2 overexpression under the SPI1 promoter did not interfere with growth (which was slightly faster than for the wild-type strain). Cells reached a similar final density, but viability remained higher than the parental strain after sugar depletion. If maximal cell viability (in this case, day 8 for the wild-type strain) was taken as $100 \%$ survival, a plot showing the death profile was obtained (Figure 1C). In this case, it can be clearly seen that the overexpression of SIR2 extended CLS under winemaking conditions, which is the exact opposite effect to that produced by SIR2 deletion [28]. The SIR2 expression under the MET17 promoter slightly reduced final cell density (Figure 1B), but also extended CLS (Figure 1C). This result indicates that Sir2 plays a positive role on CLS under winemaking conditions, which is the equivalent to the positive role on RLS under laboratory conditions [35]. Therefore, despite the controversial role in aging of the Sir2 overexpression in animal models [27], it plays a role in longevity in S. cerevisiae as similar results have been obtained in different genetic backgrounds and experimental conditions.

We performed the same overexpression strategy on another member of the sirtuin family, HST3, whose deletion had the opposite effect to SIR2 deletion in CLS and ethanol production [28]. Its deletion led to an increase in CLS and a slightly smaller amount of ethanol. The survival curve (similar to that described in Figure 1C) under fermentation conditions showed that the overexpression of HST3 with both promoters leads to a slightly shortened CLS (Figure 1D), the opposite effect to that observed for the SIR2 overexpression, and is also the reverse effect to that induced by HST3 deletion. Fermentation capacity did not alter (data not shown); therefore, wine yeast chronological longevity under winemaking conditions can be modulated by the deletion or overexpression of different sirtuin family members.

Next we overexpressed the gene coding for acetyltransferase Gcn 5 under the control of the MET17 promoter, as it gives high expression through all fermentation (see Figure 1A). GCN5 deletion caused CLS to extend [28] and, unexpectedly, its up-regulation also extended maximal life span (Figure 1E). As Gcn5 acts with a variety of multiprotein complexes, such as SAGA, SLIK or SALSA [36], its overexpression may alter the amount or proportion of these complexes, thus 


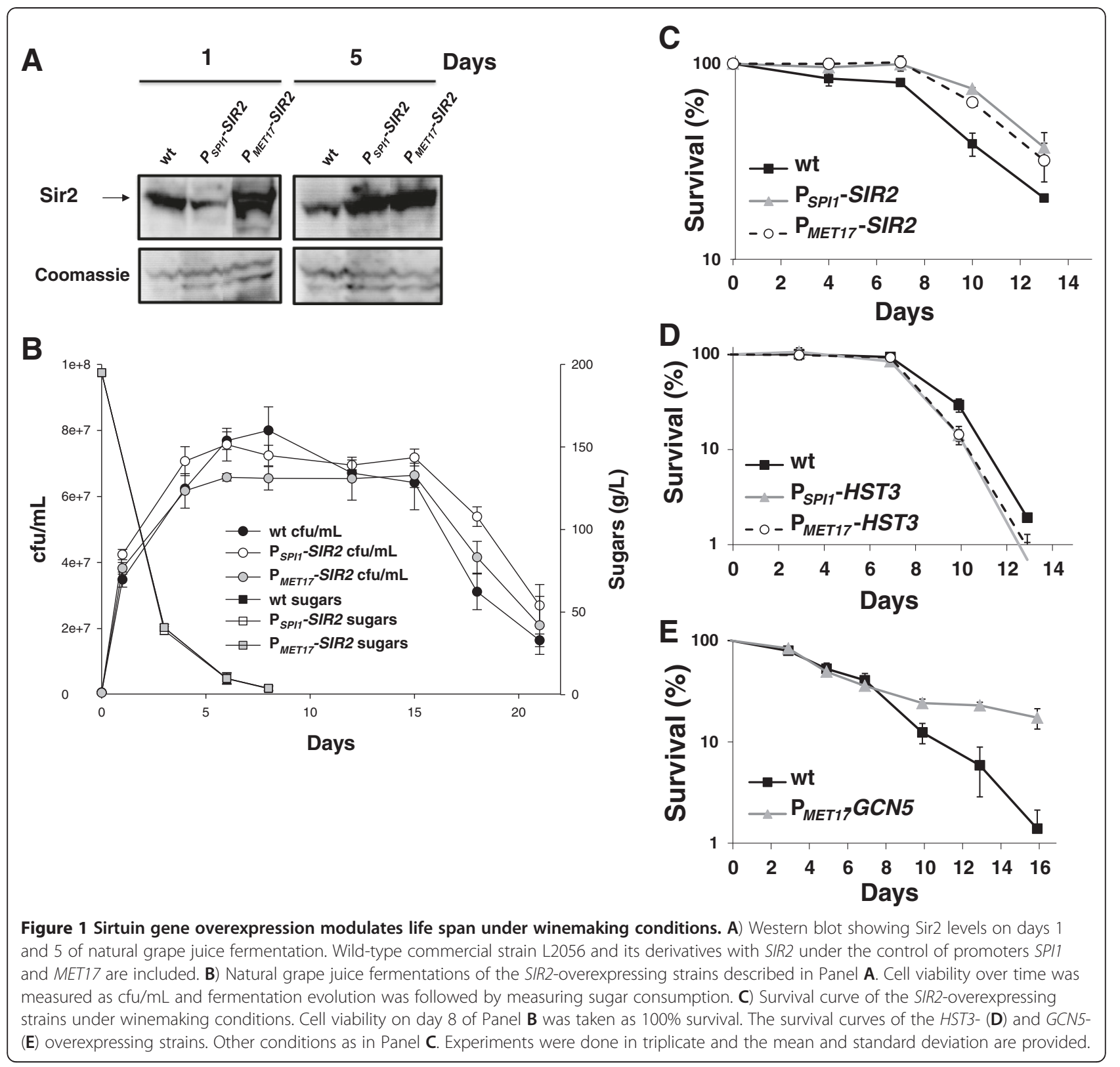

producing this unexpected impact on longevity as they regulate multiple targets.

\section{HST3 overexpression increases ethanol production and tolerance}

Our next objective was to test the effect of these overexpressions on metabolite production at the end of grape juice fermentation (Figure 2). As mentioned earlier, none of these manipulations altered the ability to fully consume sugars under winemaking conditions. We measured three essential metabolites for wine organoleptic properties: ethanol, acetic acid and glycerol. Regarding ethanol production (Figure 2A), only HST3 overexpression under the MET17 promoter significantly increased ethanol production. When the HST3 overexpression was prompted by the SPI1 promoter, it led to a slight, but not significant, increase in final ethanol production. This result matches the fact that HST3 deletion reduces ethanol production [28], possibly indicating that ethanol accumulation causes premature aging in these strains (Figure 1C). However, SIR2 manipulations had no impact on ethanol production, despite the role of the corresponding deletion on ethanol metabolism [28]. To test if the increase of ethanol production in HST3-overexpressing strains altered biomass production, we measured the dry biomass after completion of fermentation 


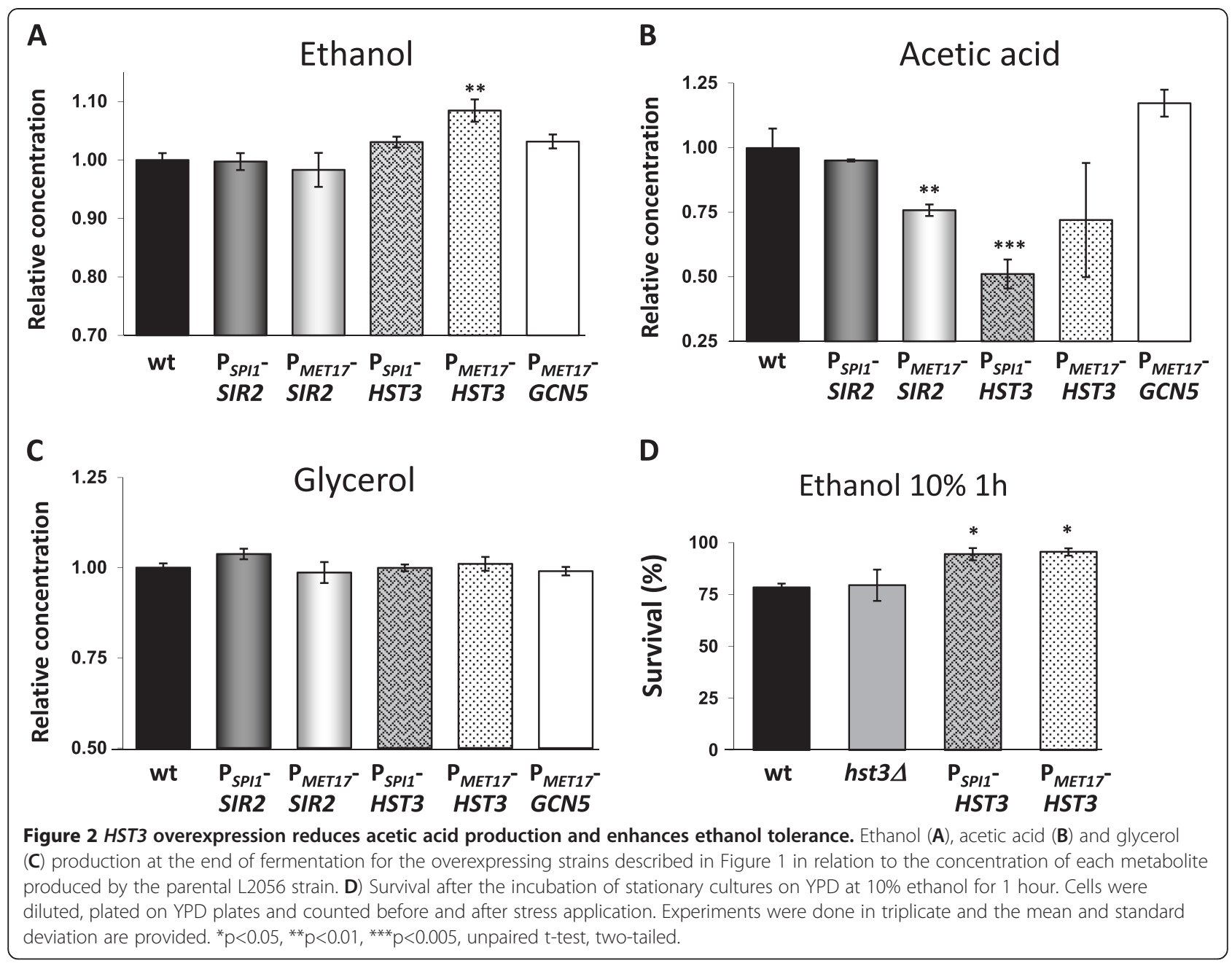

carried out in synthetic grape juice MS300 (to minimize the effect of solids present in natural grape juice). Both overexpressing strains had a decreased biomass production (Additional file 1: Figure S1A). Ethanol is also increased in this medium, particularly for the SPI1 promoter-driven HST3 mutant (Additional file 1: Figure S1B). Therefore HST3 activity cause a shift of carbon from biomass production to ethanol production.

Acetic acid production was also affected, as expected, by HST3 overexpression (Figure 2B). HST3 deletion increased acetic acid production [28], and its overexpression brought about a lower final concentration (although it was significant only in the strain with the SPI1 promoter-driven HST3). A lower acetic acid level did not correlate with the shortened longevity of those strains. Therefore, acetic acid is not the main cause of CLS under winemaking conditions. SIR2 deletion has been described to cause low acetic acid production, and its overexpression by the MET17 promoter surprisingly led to a similar phenotype (Figure 2B). It is also striking that GCN5 overexpression caused no major impact on acetic acid production, (only a slight increase was seen), while its deletion notably increased acetic acid production under several growth conditions [28]. Likely, the multiple targets of those proteins may explain the complex metabolite profile produced by their overexpression, that do not always have the opposite output than their deletion.

As regards glycerol production (Figure 2C), lack of effects contrasts with the increased glycerol production observed in the $g c n 5 \Delta$ strain [28]. In fact, none of these genetic alterations was able to significantly change the amount of this relevant metabolite. It is also worth mentioning that none of these genetic manipulations alter acetaldehyde production (data not shown). Therefore the impact of acetylation/deacetylation machinery in metabolite production is complex and difficult to predict, but can highlight interesting strategies to change the metabolic profile of wine, as the case of HST3 overexpression proves. Although we do not yet have a molecular explanation for the role of Hst3 in metabolic regulation, the combined deletion of HST3 and its 
homolog HST4 in laboratory strains prevents growth on acetate and propionate as a sole carbon source [37], suggesting that yeast sirtuins may act as human or Salmonella sirtuins to modulate the action of acetylCoA synthetase (Acs). However, there is no evidence of a reversible acetylation involved in Acs regulation, and we detected no change in Acs activity in the hst $3 \Delta$ or hst $4 \Delta$ mutants [28], so the implication of sirtuins in this regulation may be redundant.

Changes in ethanol production may affect yeast cells' ability to deal with toxicity caused by an accumulation of this metabolite, the main stress condition at the end of wine fermentation. Deletion of sirtuins, including HST3, had no impact on ethanol tolerance (Figure 2D and data not shown), but HST3 overexpression not only increased ethanol production (Figure $2 \mathrm{~A}$ ), but also enhanced tolerance to it (Figure 2D). Despite increased tolerance, HST3 overexpression shortened CLS (Figure 1D); therefore, an increase in ethanol may be detrimental for CLS, even for these stress-resistance strains. HST3 transcription was induced by cocoa polyphenols and proved necessary for the protection that these polyphenols offer against oxidative stress [38]. Therefore, sirtuin manipulation could allow to modulate stress tolerance in yeast under natural conditions.

\section{Superoxide dismutase SOD2 overexpression has a limited} impact on life span during winemaking

According to the free radical theory of aging, the induction of anti-oxidant enzymes should contribute to extend life span. One of these enzymes is mitochondrial superoxide dismutase Sod2, which performs an important task in CLS under laboratory conditions [39]. To test the impact of improving oxidative stress tolerance at the stationary phase on winemaking conditions, we expressed the SOD2 gene under stationary phase promoter SPI1 in wine yeast L2056. This transformation did not significantly affect grape juice fermentation as sugar consumption profiles were similar for modified and parental strains (Figure 3). Cell growth between strains was also similar, but cell viability increased in the overproducing strain when sugar levels were low. However when sugars were exhausted, both strains showed a similar long-term life span. Therefore, the impact of mitochondrial defense systems on longevity does not seem relevant when sugars are absent, thus mitochondrial activity is assumed to be not glucose-repressed. This indicates that this cellular metabolic situation differs from the stationary phase in standard growth media for laboratory yeast strains. SOD2 overexpression brought about the opposite effect to the deletion of another mitochondrial antioxidant gene, $R D L 2$ [40]. In this case, viability before glucose exhaustion dropped and ROS concentration increased. Therefore, these results suggest that mitochondrial activity and anti-oxidant responses are necessary at the end of fermentation when the sugar concentration is low. Yet surprisingly once fermentation has finished, these systems seem to play no role, despite the fact that the absence of sugars is no longer repressing respiration and stress response. It should be taken into account that ethanol is not consumed at the end of vinification, therefore our molecular results indicate that this physiological situation is not the equivalent to that of laboratory strains growing on a non-fermentable carbon source.

\section{RNA-binding proteins are life span modulators under winemaking conditions}

Next we focused on a different family of proteins with an impact on longevity, RNA-binding proteins, which

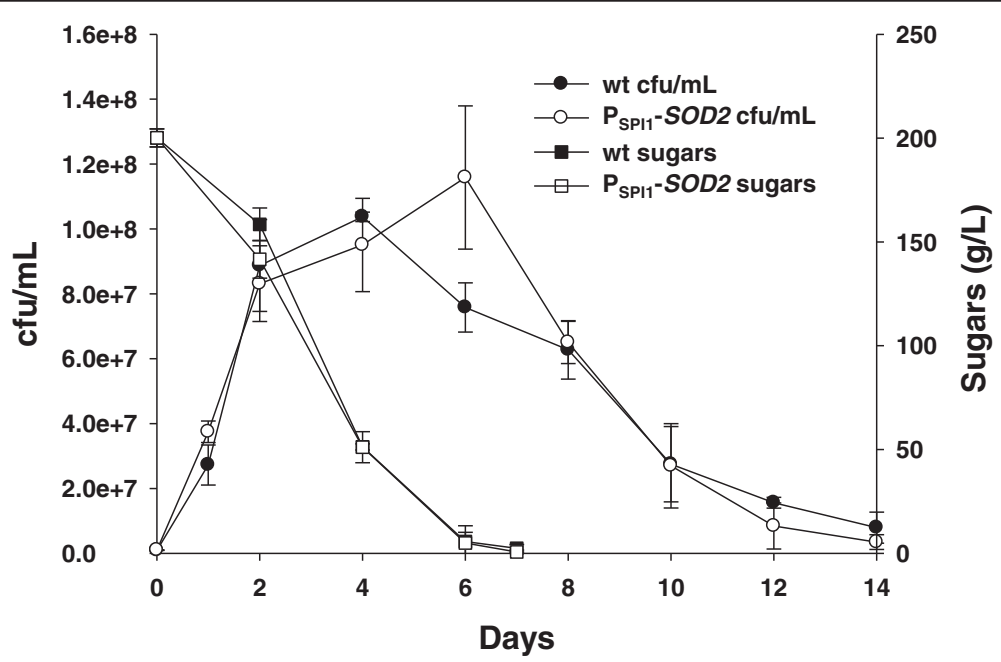

Figure 3 Superoxide dismutase 2 overexpression has a mild impact on life span. The SOD2-overexpressing strain and its parental strain L2056 were used to inoculate natural grape juice fermentations. Cell viability over time was measured as cfu/mL and fermentation evolution was followed by measuring sugar consumption. Experiments were done in triplicate and the mean and standard deviation are provided. 
may control many different processes at the posttranscriptional level. Uth4/Puf5 was one of the first agerelated genes to be described [15]. In a global analysis of CLS, Puf3 (an Uth4 homolog) and Pub1 were two RNA binding proteins whose deletion increased life span [41]. Ngr1 was another RNA binding protein seen to be required for stationary phase survival [42]. We deleted all four genes in haploid wine strain C9 [43]. First, we tested the effect of the deletions on CLS under standard laboratory conditions in SC medium (Figure 4A). NGR1 mutation brought about a sharp drop in viability, indicating its positive role in CLS in wine yeast. Uth4, which is required for replicative life span under laboratory strains, was also required to achieve full CLS in wine yeasts. In accordance with what happened in laboratory strains, the PUB1 deletion extended CLS. However, the PUF3 deletion, that in some experiments extends CLS [41] and in other does not affect it [44], unexpectedly shortened it (Figure 4A). That suggest an influence of genetic background on Puf3 function. Puf3 controls the levels of the mRNAs coding for mitochondrial proteins, suggesting that mitochondria may act in S. cerevisiae laboratory and wine strains in different ways.

The same strains were used to conduct fermentations on natural red grape juice. All the mutants completed fermentation successfully (Additional file 2: Figure S2A). The death profile at the end of fermentation (as seen in Figure $1 \mathrm{~B}$ ) is shown in Figure $4 \mathrm{~B}$. Consistently with the results obtained in the laboratory medium, NGR1 and UTH4 deletions shortened CLS, while deletion PUB1 slightly prolonged it. However, the effect of PUF3 deletion changed depending on the medium as its deletion lengthened CLS in grape juice (Figure 4B), which is the opposite effect seen in SC medium, but the same effect described for laboratory strains [41]. Due to the role of Puf3 in mitochondrial function regulation, the low oxygen level during grape juice fermentation must impose different conditions to mitochondria than the growth in low glucose in laboratory media, where respiration is necessary for longevity. Metabolites were monitored at the end of fermentation (Figures $4 \mathrm{C}, \mathrm{D}, \mathrm{E})$. Ethanol production did not change significantly (Figure 4C), but the

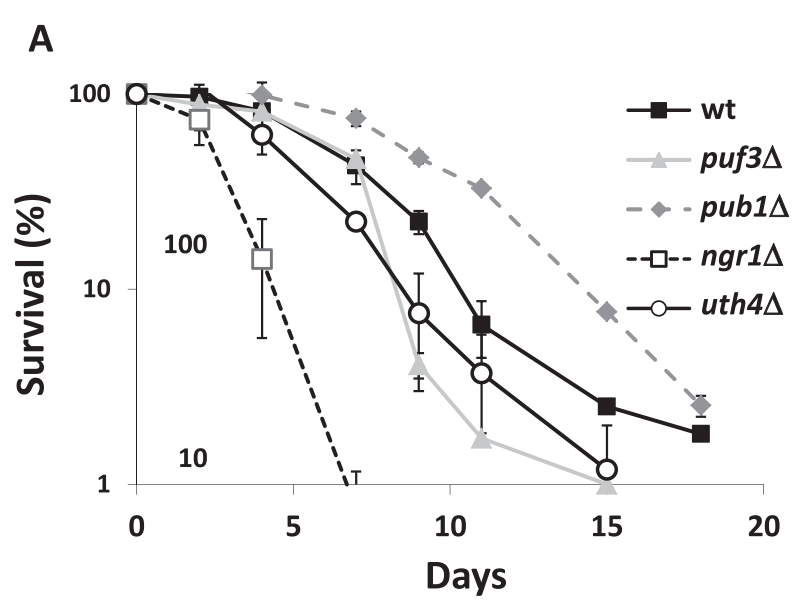

C

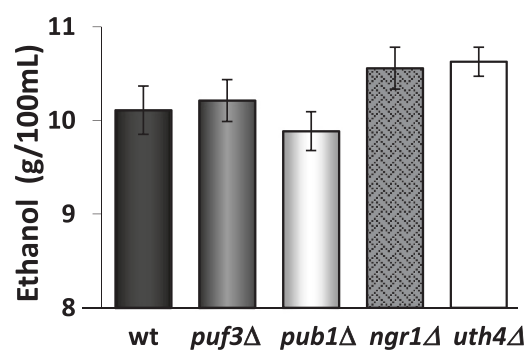

D

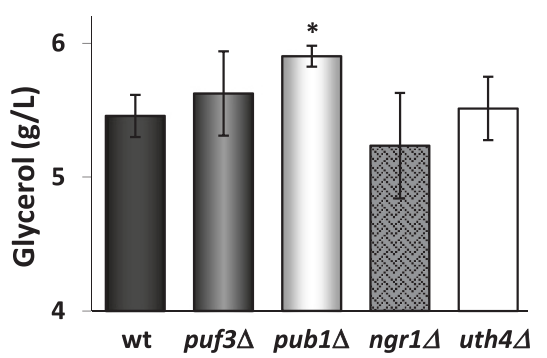

B

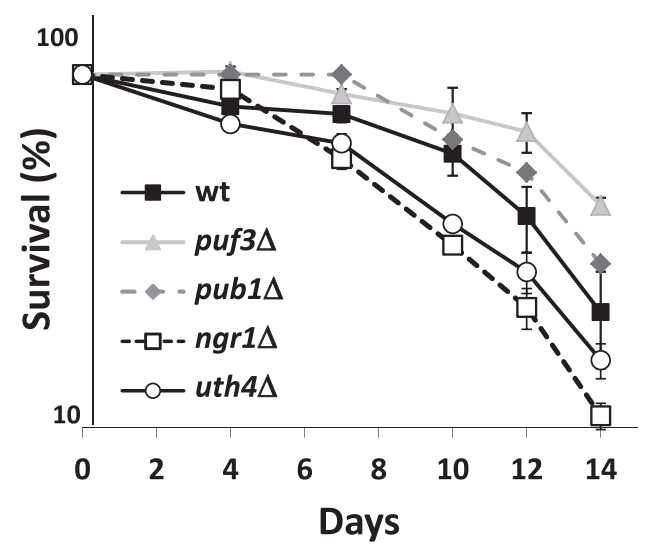

E

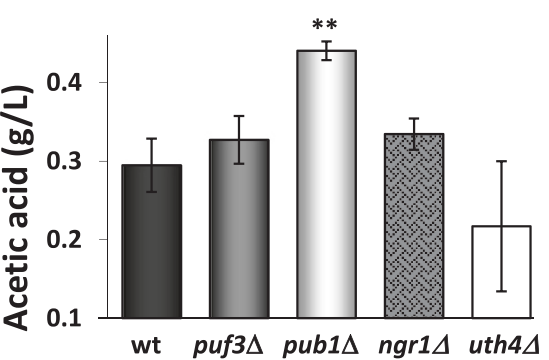

Figure 4 Deletion of mRNA binding proteins alters lifespan under different growth conditions. Genes were deleted from haploid wine yeast C9. A) The CLS experiments in laboratory medium SC. Viability after three days in SC is taken as $100 \%$ survival. Aliquots of each culture were taken, diluted, plated and counted over time. B) The CLS plots during the grape juice fermentation of the same deletion strains. Other conditions as in Figure 1C. Production of ethanol (C), acetic acid (D) and glycerol $(\mathbf{E})$ in the vinifications described in Panel (B). Experiments were done in triplicate and the mean and standard deviation are provided. ${ }^{*} p<0.05,{ }^{*} p<0.01$, unpaired t-test, two-tailed. 
PUB1 deletion increased glycerol (Figure 4D) and acetic acid (Figure 4E). Pub1 binds the mRNA of glycerol 3-phosphate dehydrogenase GPD2 [45], which could contribute to this increased production.

As the PUB1 deletion extended longevity in a variety of growth media and it increased the levels of glycerol, a metabolite with a positive impact on wine properties, we performed the deletion of the two copies of PUB1 present in industrial wine strain EC1118, a widely used commercial strain with a relatively short CLS if compared to other strains [12]. The null pub1s mutant was able to complete natural grape juice fermentation (Figure 5A), although the sugar consumption rate was slightly slower at later fermentation stages. Cell growth was normal and life span extended once sugars had been exhausted in the deletion mutant, as it happened in the previous experiments. Metabolite production at the end of this fermentation was measured (Figures 5B,C,D). Ethanol and acetic acid production were mildly lower in the mutant strain, but in this case, the drop in acetic acid was not significant (Figure 5C), unlike what happened in the C9 strain. Therefore, genetic background influenced the output of this mutation. However, glycerol production was significantly higher in the strain carrying the PUB1 deletion (Figure 5D), as was the case in the C9 background, indicating that Pub1 plays a relevant role in glycerol production, and that its manipulation is an efficient strategy to increase the concentration of this valuable metabolite.

Pub1 and Ngr1 take part in cytoplasmic aggregates known as stress granules (SG) during glucose starvation and other stress conditions [46]. SG contain mRNA and translation factors, such as the poly(A) binding protein Pab1. In fact, the PUB1 deletion blocks the formation of such SG in glucose starvation [47], but not in the presence of translation inhibitor sodium azide [48]. However NGR1 deletion has no effect in SG formation [47]. We tagged with GFP the SG specific protein Pab1 in wine yeast EC1118 and its pub1s derivative, and SG formation was followed during grape juice fermentation (Figure 6A). Pab1 has a uniform cytosolic distribution during the first days of fermentation, and SG do not appear in the wild type strain up to day four, when cells reached stationary phase and growth ceased (see Figure 5A). In this conditions, SG are not formed in the mutant strain (Figure 6A). At day 7, when fermentation is finished, SG are bigger in the wild type strain, but in the pub1s strain they are absent or very faint. Therefore, stress granules are formed in the late phases of winemaking, and their formation depends on Pub1. Next we observed SG formation in the
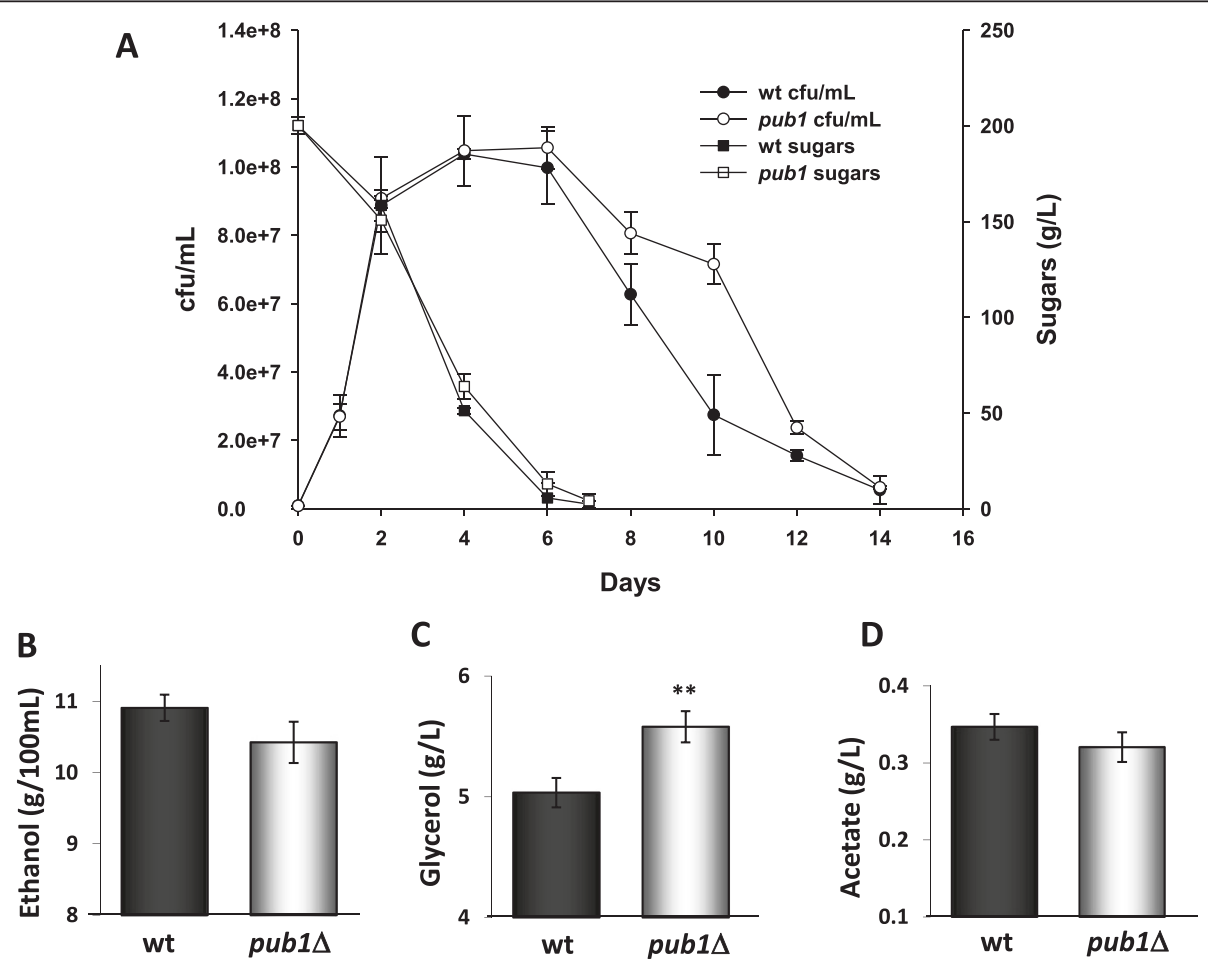

Figure 5 The PUB1 deletion increases glycerol production and extends CLS during winemaking. A) Natural grape juice fermentations of the PUB1 deletion strain and its parental strain L2056. Cell viability over time was measured as cfu/mL and fermentation evolution was followed by measuring sugar consumption. Ethanol (B), acetic acid (C) and glycerol (D) production of the fermentation described in Panel $\mathbf{A}$. Experiments were done in triplicate and the mean and standard deviation are provided. ${ }^{* *} p<0.01$, unpaired t-test, two-tailed. 


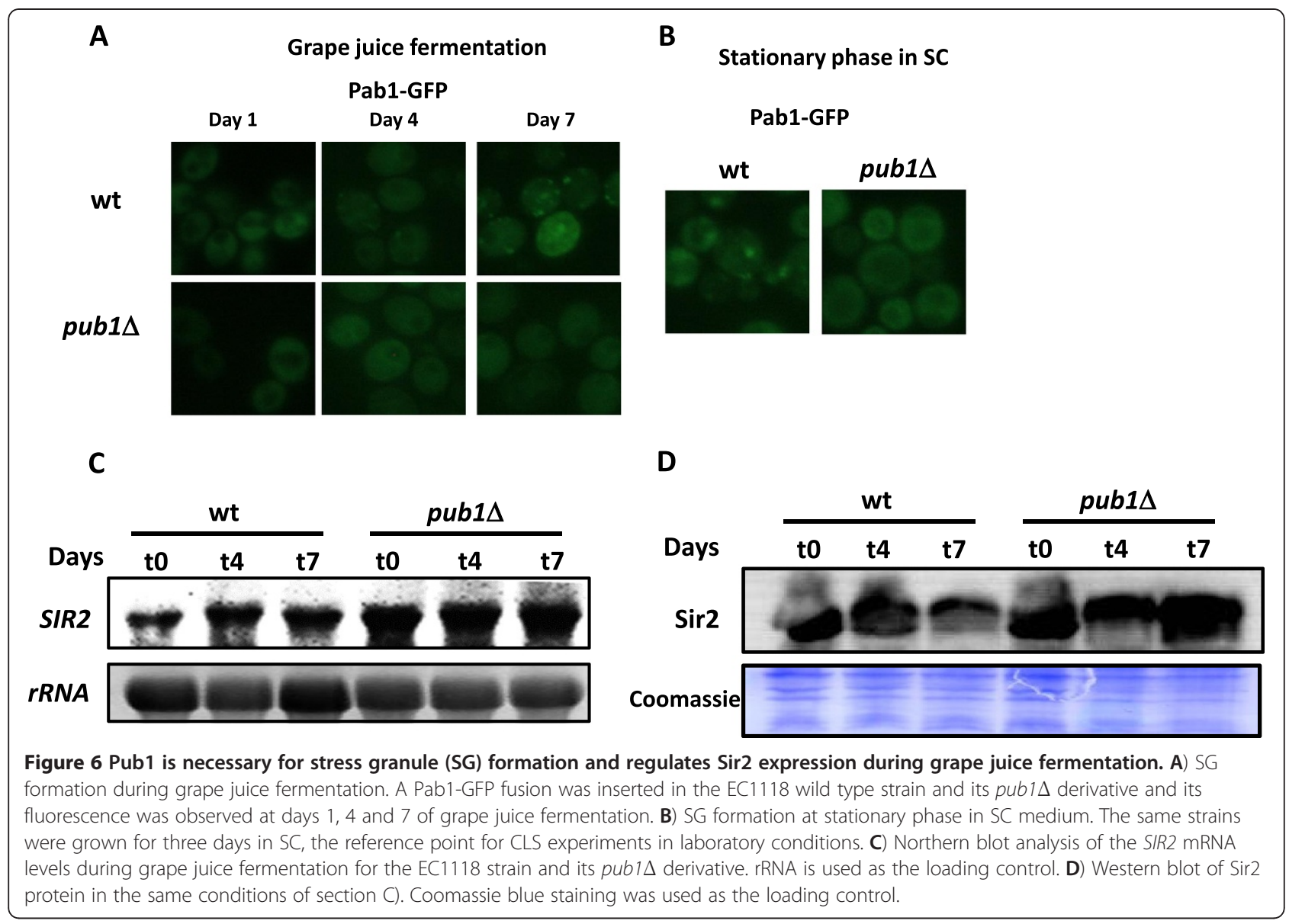

laboratory medium SC where CLS experiments are performed. In stationary phase after three days in this medium (that correspond to day 0 in Figure 4A), stress granules are formed (Figure 6B), although they tend to be fewer (many times there is only one per cell) than the ones observed in grape juice fermentation. PUB1 deletion cause also SG disruption in this condition. Therefore, stress granule formation is not necessary to achieve full life span, and they may be even deleterious for longevity, as PUB1 deletion extends CLS and reduce SG in both conditions tested. Maybe SG are important to survival after short stresses but sequestration of translation factors may impair long-term survival.

Pub1 is also a gene expression regulator that has been linked to the control of mRNA stability and translation [45]. We have previously seen that SIR2 mRNA levels change when cells are exposed to acetaldehyde, suggesting that they are controlled by the metabolic status of the cell [49]. RNA was extracted during grape juice fermentation on days 1, 4 and 7, and the level of SIR2 mRNA was measured by Northern blot (Figure 6C). We saw that SIR2 was up-regulated in the pub1D mutant throughout fermentation. In a global analysis, SIR2 mRNA was not detected as a binding target of the Pub1 protein [45], so the effect of Pub1 on SIR2 mRNA levels may be indirect. The deletion of other mRNA binding proteins had no impact on the SIR2 mRNA levels (data not shown). We measured Sir2 protein levels during fermentation by western blot (Figure 6D) and found that protein levels are also higher in the PUB1 deletion strain, in similar levels, suggesting that most of the effect of Pub1 on SIR2 gene expression is acting at the RNA level, not at translation, as protein levels follow mRNA abundance. In any case that elevated Sir2 levels may contribute to extend longevity during grape juice fermentation in the PUB1 deletion strain.

\section{Apoptosis plays an unexpected positive role during winemaking}

Finally, we tested the effect of the deletion of apoptosisrelated genes during grape juice fermentation. We chose yeast caspase gene $Y C A 1$ and mitochondria-related apoptosis-inducing factor AIF1 to test different branches of the process. Both deletions extended CLS under laboratory conditions [50,51]. We performed independent and double deletions in haploid wine yeast strain C9 to construct the double mutant $y c a 1 \Delta$ aif $1 \Delta$ easily. These strains were used as starters during natural grape juice 


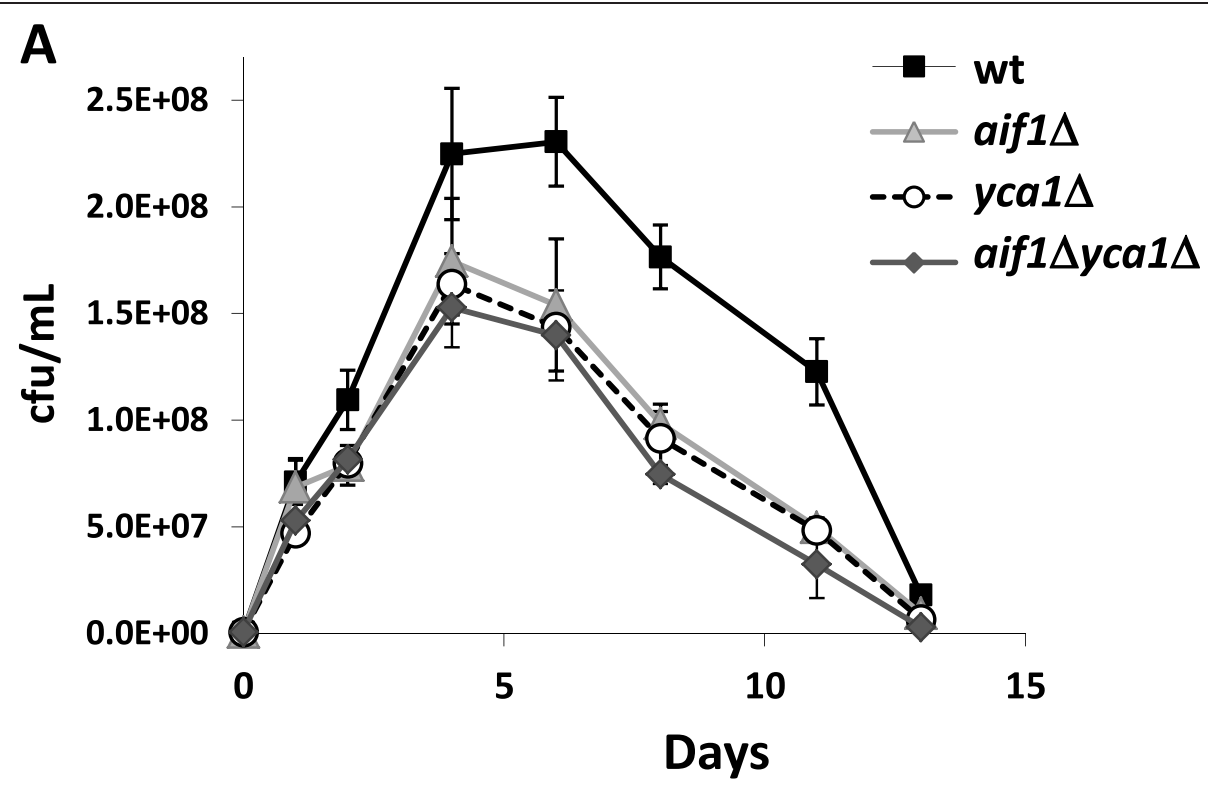

B

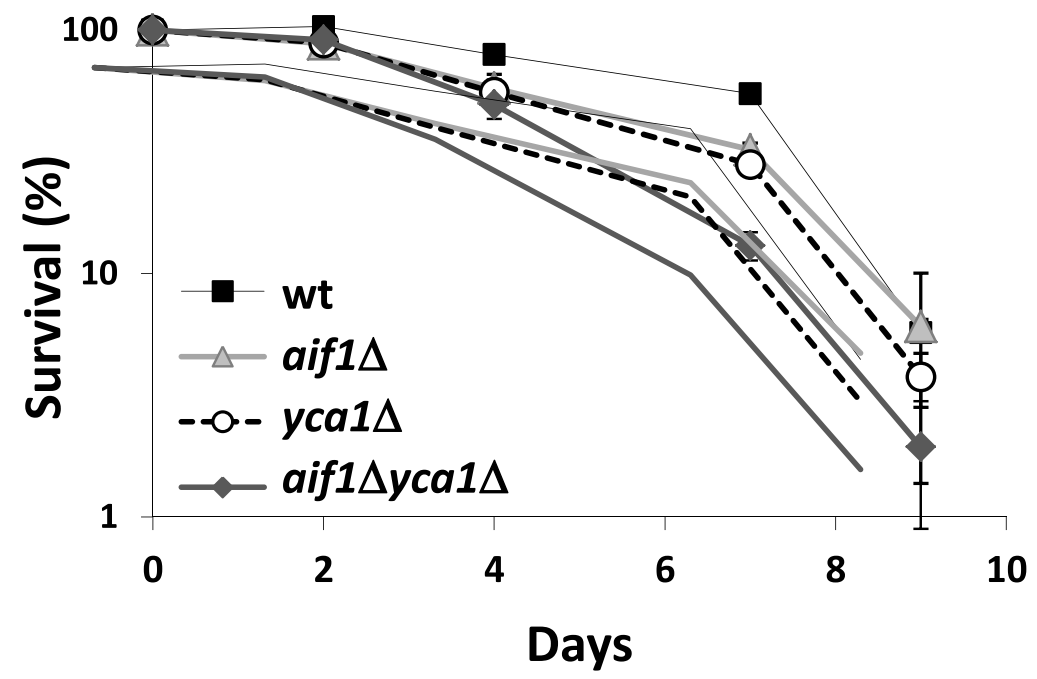

C

PI

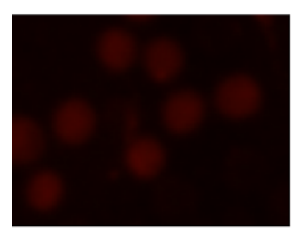

DAPI

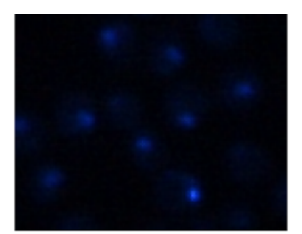

Merged

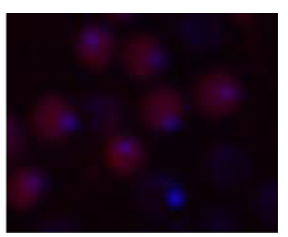

Figure 7 Deletion of apoptosis-related genes shortens CLS under winemaking conditions. The deletion mutants of YCA 1, AIF1, or both, on the C9 strain were used for grape juice fermentations. A) Cell viability over time was measured as cfu/mL. B) Survival curves after taking day 4 of fermentation as 100\% survival. Experiments were done in triplicate and the mean and standard deviation are provided. C) C9 strain after 8 days of fermentation was co-stained with propidium iodide (PI) and DAPI, and observed under a fluorescence microscopy. A merged image is also shown. 
fermentation. Mutants were able to complete fermentation and showed no defect upon sugar consumption (Additional file 2: Figure S2B). The growth profiles of all strains are shown in Figure 7A. All the mutant strains presented a slower cell growth rate and reached lower maximal cell density. If the cell count on day 4 was taken as $100 \%$ viability, a death profile could be obtained (Figure 7B). Surprisingly, both single mutants presented a shortened life span under winemaking conditions, suggesting that both the caspase-dependent and caspaseindependent (and mitochondrial-dependent) branches of apoptosis do not promote cell death under these conditions. The fact that the double mutation effect was additive reinforces the idea that both proteins not only act in different pathways, but also play a positive role in CLS under winemaking conditions. As previously described by our group for other pathways, such as autophagy, the effect of apoptotic machinery on winemaking is the opposite to the effect observed under laboratory conditions. Growth conditions during winemaking (high sugar, low nitrogen) differ from laboratory growth conditions (low sugar, high nitrogen), which may affect the trigger of programmed death mechanisms [52]. Low oxygen condition does not seem relevant as the blockage of both mitochondrial- dependent and -independent branches of apoptosis displays similar effects under winemaking conditions.

In previous work we have observed that at the end of grape juice fermentation cells are stained by the necrosisspecific dye propidium iodide (PI) $[9,12]$. At day 8 of natural grape juice fermentation carried out by the wild type wine strain $\mathrm{C}$, death cells are stained in red by PI (Figure 7C). DAPI staining of nuclei at the same time indicate that most nuclei are intact at the end of fermentation (Figure 7C). Nuclear fragmentation is a marker of apoptosis that can be detected by DAPI staining [53]. Merged images showed that cells with damaged membrane integrity (PI stained) have intact nuclei, suggesting that cells at the end of fermentation suffer a necrotic death. Mutants in apoptosis factors showed the same profile (data not shown), reinforcing the idea that death do not happen through apoptosis in winemaking conditions.

\section{Conclusions}

In this work, we studied the effects of genetic manipulation of a variety of age-related genes under winemaking conditions. Chronological life span (CLS) during grape juice fermentation can be extended by overexpressing sirtuin gene SIR2 or by deleting RNA-binding proteins genes PUB1 and PUF3. Pub1 is necessary for stress granule formation in winemaking conditions and during standard CLS experiments in SC medium. Both sirtuin HST3 overexpression and RNA-binding protein gene NGR1 deletion increase death, therefore promoting cell lysis, which are useful processes to improve the organoleptic properties of wine or to promote malolactic bacteria growth. Aging is closely linked to metabolism, and many of those genetic manipulations lead to changes in the metabolites produced during wine fermentation. The ability of the HST3 overexpressing strain to produce high ethanol and low acetic acid is an interesting property from a commercial perspective. There is a correlation between ethanol production and CLS as an increase in the HST3 level leads to high ethanol and shortened longevity, and the strain displaying a high SIR2 expression presents increased CLS, with slightly lower ethanol production. Conversely to observations under laboratory conditions [21], acetic acid is not the main cause of aging under winemaking conditions. Glycerol has been identified as a longevity inducer under laboratory conditions [23], and may explain the longer longevity of the pub1 $1 \Delta$ strain. It is worth noting that all the manipulated strains were able to complete fermentation and that they all consumed sugars completely, and usually at a similar rate to the parental strain. Therefore, this aspect of metabolism is very robust and cells seem to adapt to changes in metabolism-related genes in order to keep the fermentation pathway at an optimal speed, although the proportion of some metabolites could change in these manipulated strains.

\section{Methods}

\section{Yeast strains and growth media}

Additional file 3: Table S1 lists the industrial wine yeasts used in this work. Haploid strain C9 was a gift from M. Walker [43]. Additional file 4: Table S2 lists the oligonucleotides employed to amplify deletion and overexpression cassettes and to check transformants. Gene over-expression strains were obtained by following a promoter replacement strategy developed in our laboratory [32]. Plasmid pkanMX-SPI1p [32] was used to PCRamplify the cassettes for gene over-expressions under the control of the SPI1 gene promoter. In order to express genes under the control of the MET17 promoter, plasmid pkanMX-MET17p was constructed by amplifying the MET17 promoter by PCR using oligonucleotides MET17a and MET17b (see Additional file 4: Table S2) and by cloning the amplified fragment at the EcoRV site of plasmid pUG6 [54]. Gene disruptions were performed by using recyclable selection marker loxP-kanMX-loxP from plasmid pUG6 following the protocol of Güldener et al. [54]. The marker was eliminated by transforming with the cre recombinase-containing plasmid YEp351cre-cyh according to Delneri et al. [55]. For GFP fusions, plasmid pFA6a-GFP(S65T)-kanMX6 [56] was used as a template to $\mathrm{C}$-terminal tagging of $P A B 1$ gene. Yeast transformations were carried out by the lithium acetate method [57]. 
For yeast growth under laboratory conditions, YPD medium ( $1 \%$ yeast extract, $2 \%$ bactopeptone, $2 \%$ glucose) and SC medium $(0.17 \%$ yeast nitrogen base, $0.5 \%$ ammonium sulfate, $2 \%$ glucose and $0.2 \%$ drop-out mix with all the amino acids) were used [58]. Selective plates contained $20 \mu \mathrm{g} \mathrm{mL} \mathrm{mL}^{-1}$ geneticine or $0.1 \mu \mathrm{g} \mathrm{mL} \mathrm{mL}^{-1}$ cycloheximide. Red grape juice (Bobal variety) was a gift from Bodegas Murviedro and was sterilized overnight with $500 \mu \mathrm{g} / \mathrm{L}$ of dimethyldicarbonate. Synthetic grape juice MS300 was made as previously described $[9,59]$.

\section{Grape juice fermentations, chronological life span measurements and stress conditions}

For the microvinification experiments, cells from the 2-day cultures in YPD were inoculated at a final concentration of $10^{6}$ cells $/ \mathrm{mL}$ in filled-in conical centrifuge tubes with $50 \mathrm{~mL}$ of grape juice. Incubation was done at very low shaking at $22^{\circ} \mathrm{C}$. Evolution of vinifications was followed by determining cell viability by diluting, plating and counting colony forming units (cfu) and sugar consumption, as previously described [60]. Survival plots were drawn by taking the highest cell viability point (around 2-4 days) as 100\% survival. Dry biomass measurement was carried out using the filtration method [61] and weighting the filters after drying at $65^{\circ} \mathrm{C}$ overnight.

Under laboratory conditions, the CLS experiments were performed as follows: precultures of selected strains were grown overnight on YPD and inoculated in $\mathrm{SC}$ media at an $\mathrm{OD}_{600}$ of 0.1. After day 3 of growth at $30^{\circ} \mathrm{C}$, aliquots were taken, diluted and plated. Colonies were counted and percentage of survival was calculated taken day 3 of growth as 100\% survival. Ethanol tolerance was measured in stationary cultures on YPD by adding $10 \%$ ethanol for $1 \mathrm{~h}$. Cell viability was measuring by diluting, plating and counting cfu.

\section{Metabolite determinations}

Consumption of sugars during fermentation was measured by their reaction to DNS (dinitro-3,5-salycilic acid) following a modified version of Miller's method [62]. Ethanol, acetic acid and glycerol were measured with the kits provided by r-Biopharm following the manufacturer's instructions.

\section{Western and Northern blots}

For the Western blot analysis, cells were broken with a volume of glass beads in a buffer containing Tris- $\mathrm{HCl}$ $0.1 \mathrm{M} \mathrm{pH} 7.5, \mathrm{NaCl} 0.5 \mathrm{M}, \mathrm{MgCl}_{2} 0.1 \mathrm{M}, \mathrm{NP} 40$ 1\% (v/v), PMSF $10 \mathrm{mM}$ and protease inhibitors (complete Mini, EDTA-free from Roche). Protein concentration was measured by the Bradford method [63] using the Bio-Rad Protein assay following the manufacturer's instructions. Extracts were diluted in loading buffer
(Tris- $\mathrm{HCl} 240 \mathrm{mM}$ pH 6.8, SDS 8\%, glycerol 40\%, $\beta$-mercaptoethanol 10\%).

To conduct the Western blot analysis, SDS-PAGE total protein separations were done in an Invitrogen mini-gel device and were blotted onto PVDF membranes. The anti-Sir2 antibodies were obtained from Santa Cruz Biotechnology (Santa Cruz, USA). The ECL Western blotting detection system (Amersham) was used following the manufacturer's instructions.

Total RNA isolation and formaldehyde denaturing agarose gels and blotting were carried out as previously described [64]. The SIR2 probe was obtained by PCR and digoxigenine labeling using the PCR DIG labelling mix from Roche according to the manufacturer's instructions. Probe detection was achieved by chemiluminescence using the DIG Northern Stater and CDPStar kits by Roche, and was detected in an LAS-1000 device by Fujifilm.

\section{Microscopy methods}

$500 \mu \mathrm{l}$ of cells were washed in PBS buffer and $5 \mu \mathrm{l}$ of a $1 \mathrm{mg} / \mathrm{mL}$ stock solution of propidium iodide and $1 \mu \mathrm{l}$ of a $1 \mathrm{mg} / \mathrm{mL}$ stock solution of DAPI (in dimethylformamide) were added to be then incubated in darkness for $20 \mathrm{~min}$. Cells were washed in PBS and visualized. GFP-labelled cells were observed directly. Cells were visualized with the right filter under a Nikon eclipse 90i fluorescence microscope.

\section{Additional files}

Additional file 1: Figure S1. Fermentation in synthetic grape juice of HST3 overproducing strains (A) Dry biomass production. (B) Ethanol production. ${ }^{*} \mathrm{p}<0.05,{ }^{* * *} \mathrm{p}<0.005$, unpaired t-test, two-tailed.

Additional file 2: Figure S2. Sugar consumption during grape juice fermentation by mutants in mRNA binding proteins (A) and apoptosis related genes (B). The data reflect the experiments shown in Figure $4 B$ and Figure 7A respectively.

Additional file 3: Table S1. Yeast strains used in this work.

Additional file 4: Table S2. Oligonucleotides used in this work.

\section{Competing interests}

The authors declare that they have no competing interests.

\section{Authors' contributions}

$\mathrm{HO}$ carried out the experimental methods and analyzed the data. EM contributed to the experimental design. AA designed the experiments and wrote the manuscript. All the authors discussed the data, and read, reviewed and approved the final manuscript.

\section{Acknowledgments}

This work has been funded by grants from the Spanish Ministry of Science (AGL2008-00060 and AGL2011-24353) and CSIC (PIE 200970I028). H.O. is supported by an F.P.I. fellowship. We acknowledge support of the publication fee by the CSIC Open Access Publication Support Initiative through its Unit of Information Resources for Research (URICI). 


\section{Author details}

'Departamento de Biotecnología, Instituto de Agroquímica y Tecnología de Alimentos-CSIC, Av. Agustín Escardino, 7, Paterna 46980, Spain. ${ }^{2}$ Departament de Bioquímica i Biologia Molecular, Universitat de València, Avda. Dr Moliner 50, Burjassot 46100, Spain.

Received: 14 August 2012 Accepted: 27 December 2012

Published: 2 January 2013

\section{References}

1. Carnes BA: What is lifespan regulation and why does it exist? Biogerontology 2011, 12:367-374.

2. Kaeberlein M: Lessons on longevity from budding yeast. Nature 2010, 464:513-519.

3. Powell CD, Van Zandycke SM, Quain DE, Smart KA: Replicative ageing and senescence in Saccharomyces cerevisiae and the impact on brewing fermentations. Microbiology 2000, 146(Pt 5):1023-1034.

4. Basso LC, de Amorim HV, de Oliveira AJ, Lopes ML: Yeast selection for fuel ethanol production in Brazil. FEMS Yeast Res 2008, 8:1155-1163.

5. Ribéreau-Gayon P, Dubourdieu D, Donèche B: Handbook of enology. 2nd edition. Chichester, West Sussex, England; Hoboken, NJ: John Wiley; 2006.

6. Qin H, Lu M: Natural variation in replicative and chronological life spans of Saccharomyces cerevisiae. Exp Gerontol 2006, 41:448-456.

7. Fornairon-Bonnefond C, Salmon JM: Impact of oxygen consumption by yeast lees on the autolysis phenomenon during simulation of wine aging on lees. J Agric Food Chem 2003, 51:2584-2590.

8. Alexandre H, Costello PJ, Remize F, Guzzo J, Guilloux-Benatier M Saccharomyces cerevisiae-Oenococcus oeni interactions in wine: current knowledge and perspectives. Int J Food Microbiol 2004, 93:141-154

9. Orozco H, Matallana E, Aranda A: Two-carbon metabolites, polyphenols and vitamins influence yeast chronological life span in winemaking conditions. Microb Cell Fact 2012, 11:104.

10. Harman D: Aging: a theory based on free radical and radiation chemistry. J Gerontol 1956, 11:298-300.

11. Fabrizio P, Longo VD: The chronological life span of Saccharomyces cerevisiae. Methods Mol Biol 2007, 371:89-95.

12. Orozco H, Matallana E, Aranda A: Oxidative stress tolerance, adenylate cyclase, and autophagy are key players in the chronological life span of saccharomyces cerevisiae during winemaking. Appl Environ Microbiol 2012, 78:2748-2757

13. Blagosklonny MV: Aging: ROS or TOR. Cell Cycle 2008, 7:3344-3354.

14. Kennedy BK, Austriaco NR Jr, Zhang J, Guarente L: Mutation in the silencing gene SIR4 can delay aging in S. cerevisiae. Cell 1995, 80:485-496

15. Kennedy BK, Gotta M, Sinclair DA, Mills K, McNabb DS, Murthy M, Pak SM, Laroche T, Gasser SM, Guarente L: Redistribution of silencing proteins from telomeres to the nucleolus is associated with extension of life span in S. cerevisiae. Cell 1997, 89:381-391.

16. Longo VD, Kennedy BK: Sirtuins in aging and age-related disease. Cell 2006, 126:257-268

17. Dang W, Steffen KK, Perry R, Dorsey JA, Johnson FB, Shilatifard A, Kaeberlein M, Kennedy BK, Berger SL: Histone $\mathrm{H} 4$ lysine 16 acetylation regulates cellular lifespan. Nature 2009, 459:802-807.

18. Fabrizio P, Gattazzo C, Battistella L, Wei M, Cheng C, McGrew K, Longo VD: Sir2 blocks extreme life-span extension. Cell 2005, 123:655-667.

19. Lin YY, Lu JY, Zhang J, Walter W, Dang W, Wan J, Tao SC, Qian J, Zhao Y, Boeke JD, et al: Protein acetylation microarray reveals that NuA4 controls key metabolic target regulating gluconeogenesis. Cell 2009, 136:1073-1084.

20. Ralser M, Michel S, Breitenbach M: Sirtuins as regulators of the yeast metabolic network. Front Pharmacol 2012, 3:32.

21. Burtner CR, Murakami CJ, Kennedy BK, Kaeberlein M: A molecular mechanism of chronological aging in yeast. Cell Cycle 2009, 8:1256-1270.

22. Murakami CJ, Wall V, Basisty N, Kaeberlein M: Composition and acidification of the culture medium influences chronological aging similarly in vineyard and laboratory yeast. PLoS One 2011, 6:e24530.

23. Wei M, Fabrizio P, Madia F, Hu J, Ge H, Li LM, Longo VD: Tor1/Sch9regulated carbon source substitution is as effective as calorie restriction in life span extension. PLOS Genet 2009, 5:e1000467.

24. Fontana $L$, Partridge $L$, Longo VD: Extending healthy life span-from yeast to humans. Science 2010, 328:321-326.
25. Lin SJ, Defossez PA, Guarente L: Requirement of NAD and SIR2 for lifespan extension by calorie restriction in Saccharomyces cerevisiae. Science 2000, 289:2126-2128.

26. Couzin-Frankel J: Genetics. Aging genes: the sirtuin story unravels. Science 2011, 334:1194-1198.

27. Burnett C, Valentini S, Cabreiro F, Goss M, Somogyvari M, Piper MD, Hoddinott M, Sutphin GL, Leko V, McElwee JJ, et al: Absence of effects of Sir2 overexpression on lifespan in C. elegans and Drosophila. Nature 2011, 477:482-485.

28. Orozco H, Matallana E, Aranda A: Wine yeast sirtuins and Gcn5p control aging and metabolism in a natural growth medium. Mech Ageing Dev 2012, 133:348-358.

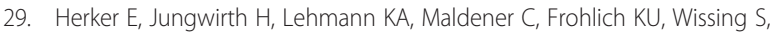
Buttner S, Fehr M, Sigrist S, Madeo F: Chronological aging leads to apoptosis in yeast. J Cell Biol 2004, 164:501-507.

30. Fabrizio P, Battistella L, Vardavas R, Gattazzo C, Liou LL, Diaspro A, Dossen $J W$, Gralla EB, Longo VD: Superoxide is a mediator of an altruistic aging program in Saccharomyces cerevisiae. J Cell Biol 2004, 166:1055-1067.

31. Ruckenstuhl C, Carmona-Gutierrez D, Madeo F: The sweet taste of death: glucose triggers apoptosis during yeast chronological aging. Aging (Albany NY) 2010, 2:643-649.

32. Cardona F, Carrasco P, Perez-Ortin JE, del Olmo M, Aranda A: A novel approach for the improvement of stress resistance in wine yeasts. Int J Food Microbiol 2007, 114:83-91.

33. Rossignol T, Dulau L, Julien A, Blondin B: Genome-wide monitoring of wine yeast gene expression during alcoholic fermentation. Yeast 2003, 20:1369-1385

34. Puig S, Perez-Ortin JE: Stress response and expression patterns in wine fermentations of yeast genes induced at the diauxic shift. Yeast 2000, 16:139-148.

35. Kaeberlein M, McVey M, Guarente L: The SIR2/3/4 complex and SIR2 alone promote longevity in Saccharomyces cerevisiae by two different mechanisms. Genes Dev 1999, 13:2570-2580.

36. Koutelou E, Hirsch CL, Dent SY: Multiple faces of the SAGA complex. Curr Opin Cell Biol 2010, 22:374-382

37. Starai VJ, Takahashi H, Boeke JD, Escalante-Semerena JC: Short-chain fatty acid activation by acyl-coenzyme A synthetases requires SIR2 protein function in Salmonella enterica and Saccharomyces cerevisiae. Genetics 2003, 163:545-555.

38. Martorell P, Forment JV, de Llanos R, Monton F, Llopis S, Gonzalez N, Genoves S, Cienfuegos E, Monzo H, Ramon D: Use of Saccharomyces cerevisiae and Caenorhabditis elegans as model organisms to study the effect of cocoa polyphenols in the resistance to oxidative stress. J Agric Food Chem 2011, 59:2077-2085.

39. Fabrizio P, Liou LL, Moy VN, Diaspro A, Valentine JS, Gralla EB, Longo VD: SOD2 functions downstream of Sch9 to extend longevity in yeast. Genetics 2003, 163:35-46.

40. Orozco H, Matallana E, Aranda A: Oxidative stress tolerance, adenylate cyclase and autophagy are key players in yeast chronological life span during winemaking. Appl Environ Microbiol 2012, 78:2748-2757.

41. Powers RW III, Kaeberlein M, Caldwell SD, Kennedy BK, Fields S: Extension of chronological life span in yeast by decreased TOR pathway signaling. Genes Dev 2006, 20:174-184.

42. Martinez MJ, Roy S, Archuletta AB, Wentzell PD, Anna-Arriola SS, Rodriguez AL, Aragon AD, Quinones GA, Allen C, Werner-Washburne M: Genomic analysis of stationary-phase and exit in Saccharomyces cerevisiae: gene expression and identification of novel essential genes. Mol Biol Cell 2004, 15:5295-5305.

43. Walker ME, Gardner JM, Vystavelova A, McBryde C, de Barros Lopes M, Jiranek $\mathrm{V}$ : Application of the reuseable, KanMX selectable marker to industrial yeast: construction and evaluation of heterothallic wine strains of Saccharomyces cerevisiae, possessing minimal foreign DNA sequences. FEMS Yeast Res 2003, 4:339-347.

44. Chatenay-Lapointe M, Shadel GS: Repression of mitochondrial translation, respiration and a metabolic cycle-regulated gene, SLF1, by the yeast Pumilio-family protein Puf3p. PLoS One 2011, 6:e20441.

45. Duttagupta R, Tian B, Wilusz CJ, Khounh DT, Soteropoulos P, Ouyang M, Dougherty JP, Peltz SW: Global analysis of Pub1p targets reveals a coordinate control of gene expression through modulation of binding and stability. $\mathrm{Mol}$ Cell Biol 2005, 25:5499-5513.

46. Balagopal V, Parker R: Polysomes, $\mathrm{P}$ bodies and stress granules: states and fates of eukaryotic mRNAs. Curr Opin Cell Biol 2009, 21:403-408. 
47. Buchan JR, Muhlrad D, Parker R: $P$ bodies promote stress granule assembly in Saccharomyces cerevisiae. J Cell Biol 2008, 183:441-455.

48. Buchan JR, Yoon JH, Parker R: Stress-specific composition, assembly and kinetics of stress granules in Saccharomyces cerevisiae. J Cell Sci 2011, 124:228-239.

49. Aranda A, del Olmo ML: Exposure of Saccharomyces cerevisiae to acetaldehyde induces sulfur amino acid metabolism and polyamine transporter genes, which depend on Met $4 p$ and Haa $1 p$ transcription factors, respectively. Appl Environ Microbiol 2004, 70:1913-1922.

50. Madeo F, Herker E, Maldener C, Wissing S, Lachelt S, Herlan M, Fehr M, Lauber K, Sigrist SJ, Wesselborg S, Frohlich KU: A caspase-related protease regulates apoptosis in yeast. Mol Cell 2002, 9:911-917.

51. Wissing S, Ludovico P, Herker E, Buttner S, Engelhardt SM, Decker T, Link A, Proksch A, Rodrigues F, Corte-Real M, et al: An AIF orthologue regulates apoptosis in yeast. J Cell Biol 2004, 166:969-974.

52. Ring J, Sommer C, Carmona-Gutierrez D, Ruckenstuhl C, Eisenberg T, Madeo F: The metabolism beyond programmed cell death in yeast. Exp Cell Res 2012, 318:1193-1200.

53. Laun P, Pichova A, Madeo F, Fuchs J, Ellinger A, Kohlwein S, Dawes I, Frohlich KU, Breitenbach M: Aged mother cells of Saccharomyces cerevisiae show markers of oxidative stress and apoptosis. Mol Microbiol 2001, 39:1166-1173.

54. Guldener U, Heck S, Fielder T, Beinhauer J, Hegemann JH: A new efficient gene disruption cassette for repeated use in budding yeast. Nucleic Acids Res 1996, 24:2519-2524.

55. Delneri D, Tomlin GC, Wixon لـ, Hutter A, Sefton M, Louis EJ, Oliver SG: Exploring redundancy in the yeast genome: an improved strategy for use of the cre-loxP system. Gene 2000, 252:127-135.

56. Longtine MS, McKenzie A III, Demarini DJ, Shah NG, Wach A, Brachat A, Philippsen P, Pringle JR: Additional modules for versatile and economical PCR-based gene deletion and modification in Saccharomyces cerevisiae. Yeast 1998, 14:953-961.

57. Gietz RD, Woods RA: Transformation of yeast by lithium acetate/singlestranded carrier DNA/polyethylene glycol method. Methods Enzymol 2002, 350:87-96.

58. Adams A, Kaiser C, Cold Spring Harbor Laboratory: Methods in yeast genetics: a Cold Spring Harbor Laboratory course manual. 1997th edition. Plainview, N. Y: Cold Spring Harbor Laboratory Press; 1998.

59. Riou C, Nicaud JM, Barre P, Gaillardin C: Stationary-phase gene expression in Saccharomyces cerevisiae during wine fermentation. Yeast 1997, 13:903-915.

60. Zuzuarregui A, del Olmo ML: Expression of stress response genes in wine strains with different fermentative behavior. FEMS Yeast Res 2004, 4:699-710.

61. Pringle JR, Mor JR: Methods for monitoring the growth of yeast cultures and for dealing with the clumping problem. Methods Cell Biol 1975, 11:131-168

62. Robyt JF, Whelan WJ: Reducing value methods for maltodextrins. I. Chainlength dependence of alkaline 3,5-dinitrosalicylate and chain-length independence of alkaline copper. Anal Biochem 1972, 45:510-516.

63. Bradford MM: A rapid and sensitive method for the quantitation of microgram quantities of protein utilizing the principle of protein-dye binding. Anal Biochem 1976, 72:248-254.

64. Aranda A, Perez-Ortin JE, Moore C, del Olmo ML: Transcription termination downstream of the Saccharomyces cerevisiae FBP1 [changed from FPB1] poly(A) site does not depend on efficient 3'end processing. RNA 1998, 4:303-318.

doi:10.1186/1475-2859-12-1

Cite this article as: Orozco et al: Genetic manipulation of longevityrelated genes as a tool to regulate yeast life span and metabolite production during winemaking. Microbial Cell Factories 2013 12:1.

\section{Submit your next manuscript to BioMed Central and take full advantage of:}

- Convenient online submission

- Thorough peer review

- No space constraints or color figure charges

- Immediate publication on acceptance

- Inclusion in PubMed, CAS, Scopus and Google Scholar

- Research which is freely available for redistribution

Submit your manuscript at www.biomedcentral.com/submit
C Biomed Central 